\title{
The Association Between Rhabdomyolysis, Acute Kidney Injury, Renal Replacement Therapy, and Mortality
}

This article was published in the following Dove Press journal: Clinical Epidemiology

Finn Erland Nielsen (iD ${ }^{1,2}$ Johan Joakim Cordtz Thomas Bøjer Rasmussen ${ }^{3}$ Christian Fynbo Christiansen (iD) ${ }^{3}$

'Department of Emergency Medicine, Slagelse Hospital, Slagelse DK-4200, Denmark; ${ }^{2}$ Department of Emergency Medicine, Bispebjerg and Frederiksberg Hospital, Copenhagen DK-2400 NV, Denmark; ${ }^{3}$ Department of Clinical Epidemiology, Aarhus University Hospital, Aarhus N 8200, Denmark
Correspondence: Finn Erland Nielsen Department of Emergency Medicine, Bispebjerg and Frederiksberg Hospital, Ebba Lunds Vej 40A, Entrance 67. Copenhagen 2400 NV, Denmark Tel +4526822753

Fax +4538639863

Email finn.erland.nielsen@regionh.dk
Background: We examined the association between creatine phosphokinase level in rhabdomyolysis patients and risk of acute kidney injury, renal replacement therapy, and death within 30 days.

Methods: The cohort included patients admitted with rhabdomyolysis from November 1, 2011 to March 1, 2014. Rhabdomyolysis was defined as a creatine phosphokinase level higher than $1000 \mathrm{U} / \mathrm{L}$. Information on laboratory variables was obtained from a laboratory database. Medical data were obtained from registries. Acute kidney injury was defined according to the Kidney Disease Improving Global Outcome (KDIGO) guidelines. The 30-day risk of outcomes was estimated using the cumulative incidence method. Spline regression applied to imputed datasets with adjustment for baseline variables was used to assess the appropriateness of the categorization chosen for creatine phosphokinase (1000-5000 U/L, 5001-15,000 U/L, and 15,000+ U/L). Results: The study included 1027 patients $(58.2 \%$ male) with a median age of 73.5 years. The median creatine phosphokinase level at rhabdomyolysis diagnosis was $2257 \mathrm{U} / \mathrm{L}$ (interquartile range $=1404-3961 \mathrm{U} / \mathrm{L})$. The 30-day risks of acute kidney injury according to the three creatinine phosphokinase levels were $42 \%(95 \% \mathrm{CI}=38-45 \%), 44 \%(95 \% \mathrm{CI}=36-52 \%)$, and $74 \%(95 \%$ $\mathrm{CI}=57-85 \%$ ), respectively, and the risks of renal replacement therapy for the three levels were 3\% (95\% CI $=2-5 \%), 4 \%(95 \% \mathrm{CI}=2-7 \%)$, and 11\% (3-23\%), respectively. The 30-day risk of death was $17 \%(95 \% \mathrm{CI}=14-20 \%), 16 \%(95 \% \mathrm{CI}=11-22 \%)$, and $11 \%(95 \% \mathrm{CI}=3-23 \%)$, respectively. With increasing creatine phosphokinase levels, the spline plots supported the increasing risk of acute kidney injury and renal replacement therapy, as well as a decreasing risk of death. However, the risk estimates for renal replacement therapy and death were imprecise.

Conclusion: Elevated initial creatine phosphokinase values were associated with an increased risk of acute kidney injury, while estimates of the risk of renal replacement therapy and death were imprecise.

Keywords: acute kidney injury, cohort study, mortality, renal replacement therapy, rhabdomyolysis, spline plots

\section{Plain Language Summary}

Rhabdomyolysis is a condition in which muscle damage leads to accumulation of toxic muscle substances in the blood. Serious complications include acute kidney injury and death. Diagnosis of rhabdomyolysis can be difficult, and a robust method of predicting the risk of complications is lacking.

In this study we examined a group of patients with elevated levels of the enzyme creatine phosphokinase in their blood. Increased amounts of this enzyme are released into the blood when there is muscle damage. 


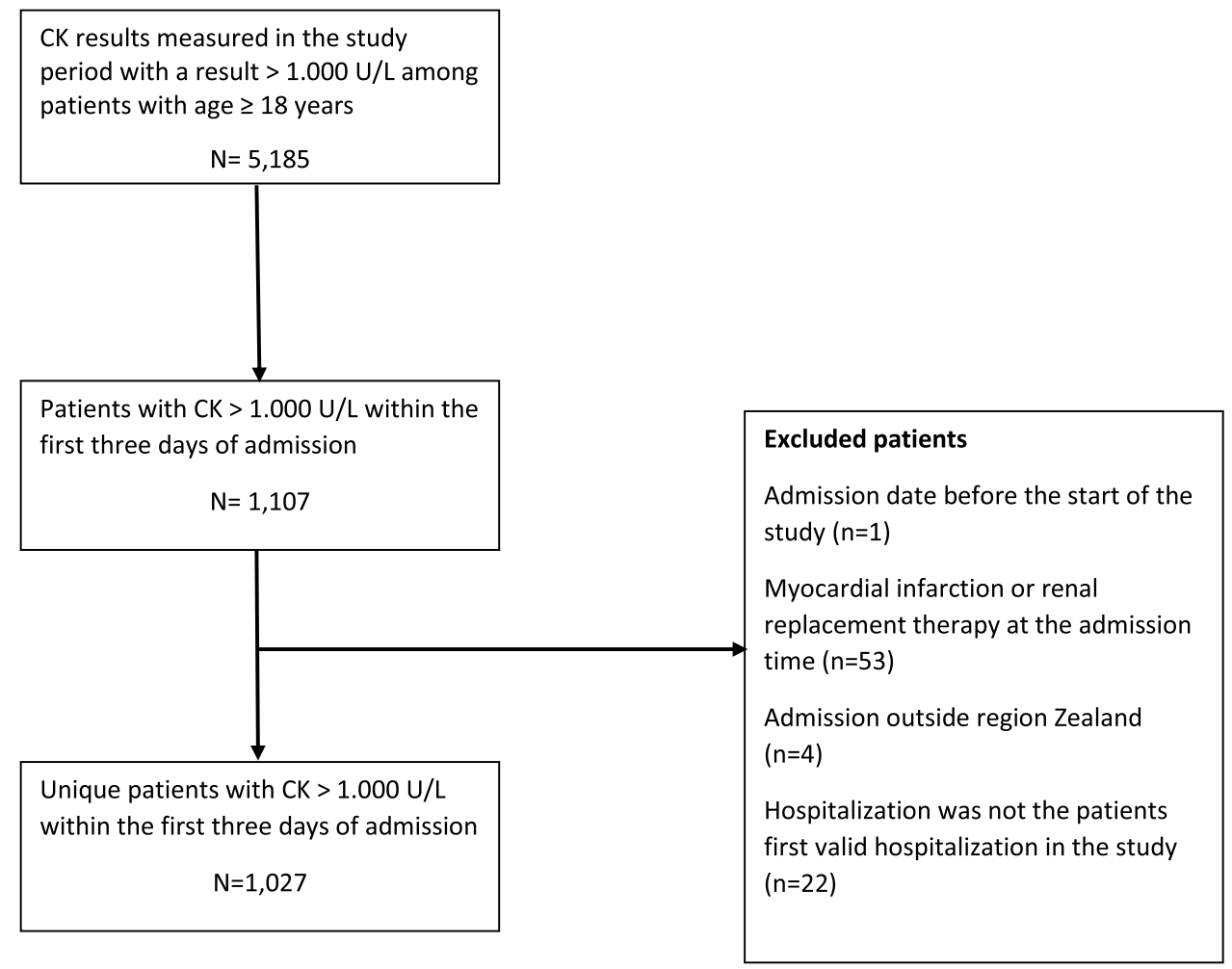

Figure I Flowchart of patients who met inclusion or exclusion criteria for the study.

We examined whether different blood levels of creatine phosphokinase are associated with an increased risk of kidney complications and death. We found an association between high levels of creatine phosphokinase and increased risk of acute kidney injury and the need for dialysis, but a lowered risk of death. We suggest that future research focus on developing kidney injury prediction models that include increased creatine phosphokinase levels as a key factor.

\section{Introduction}

Rhabdomyolysis (RM) is caused by a variety of diseases and disorders and has a wide range of symptoms, including acute kidney injury (AKI). ${ }^{1-7}$

The incidence of AKI among patients with RM has been difficult to ascertain due to a lack of accurate definitions for RM and AKI. ${ }^{6,8,9}$ Research to date suggests that the incidence ranges from $4.7 \%$ to $94 \%$ in both traumaand nontrauma-induced RM. ${ }^{6,8}$ However, due to different clinical situations, settings, small sample sizes, and confounding factors, precise estimates have been difficult to obtain. ${ }^{8}$ Consequently, further studies are needed to provide estimates of the risk of AKI among RM patients and associated mortality, as well as to delineate the determinants of AKI. The purpose of the current study was to examine the risk of $\mathrm{AKI}$, administration of renal replacement therapy (RRT) to patients with RM, and their mortality. Also, the study evaluated the association between initial levels of creatinine phosphokinase (CK) and 30-day risk of AKI, RRT, and mortality.

\section{Methods}

\section{Study Design and Setting}

We conducted this registry-based cohort study among patients admitted to any hospital in the Danish region of Zealand (approximately 830,000 inhabitants) during the period November 1, 2011 to March 1, 2014. We identified RM patients from a laboratory database covering all hospitals in the region.

\section{Rhabdomyolysis Cohort}

We identified all adult patients (aged 18 years or older) with a hospital admission and blood samples analyzed for CK, whose results were available in the laboratory database during the study period (Figure 1). All patients included in the study had CK levels $>1000 \mathrm{U} / \mathrm{L}$ within 72 hours after an admission whose indication was compatible with an RM diagnosis (index admission). ${ }^{1,2,5,7}$ Patients with preexisting renal disease treated with RRT and patients with increased $\mathrm{CK}$ levels due to acute myocardial infarction were 
Table I Baseline Characteristics in Relation to Initial Creatine Phosphokinase Level Among Patients with Rhabdomyolysis

\begin{tabular}{|c|c|c|c|c|}
\hline & $\begin{array}{l}1000-5000(U / L) \\
(N=828)\end{array}$ & $\begin{array}{l}500|-| 5,000(U / L) \\
(N=16 I)\end{array}$ & $\begin{array}{l}|5,00|+(U / L) \\
(N=38)\end{array}$ & $\begin{array}{l}\text { All Patients } \\
(\mathrm{N}=1027)\end{array}$ \\
\hline \multicolumn{5}{|l|}{ Gender } \\
\hline Female; N (\%) & $358(43.2)$ & $62(38.5)$ & $9(23.7)$ & $429(4 I .8)$ \\
\hline Age; years; median (IQR) & $74.0(61.4-83.7)$ & $71.6(57.1-80.5)$ & $64.2(53.4-79.0)$ & $73.5(60.0-83.2)$ \\
\hline \multicolumn{5}{|l|}{ Discharge diagnosis } \\
\hline Infectious diseases & III (13.4) & $37(23.0)$ & $5(13.2)$ & $153(14.9)$ \\
\hline Toxins (alcohol/medicine) & $18(2.2)$ & $5(3.1)$ & $2(5.3)$ & $25(2.4)$ \\
\hline Endocrine disorders & $62(7.5)$ & $12(7.5)$ & $6(15.8)$ & $80(7.8)$ \\
\hline Others & $637(77.0)$ & $107(66.5)$ & $25(65.8)$ & $769(74.9)$ \\
\hline $\begin{array}{l}\text { Surgery during index hospitalization, } \\
N(\%)\end{array}$ & $24 I(29.1)$ & $47(29.2)$ & $12(31.6)$ & $300(29.2)$ \\
\hline \multicolumn{5}{|l|}{$\mathrm{CCl}$ score, $\mathrm{N}(\%)$} \\
\hline 0 & $319(38.5)$ & $57(35.4)$ & $20(52.6)$ & $396(38.6)$ \\
\hline $\mathrm{I}-2$ & $327(39.5)$ & $63(39.1)$ & $12(31.6)$ & $402(39.1)$ \\
\hline $3+$ & $182(22.0)$ & $4 I(25.5)$ & $6(15.8)$ & $229(22.2)$ \\
\hline \multicolumn{5}{|l|}{ Laboratory tests; median (IQR) } \\
\hline \multicolumn{5}{|l|}{ Creatinine $(\mu \mathrm{mol} / \mathrm{L})$} \\
\hline Baseline $^{\mathrm{a}, \mathrm{b}}$ & $83.8(66.5-104.7)$ & $80.0(66.0-100.0)$ & $73.3(67.5-79.8)$ & $81.3(66.5-103.7)$ \\
\hline Upon hospital arrival & $89.0(67.0-134.0)$ & $100.5(7 \mid .0-144.5)$ & | $3 \mid .5$ (67.0-200.0) & $91.0(67.0-139.0)$ \\
\hline Calcium $(\mathrm{mmol} / \mathrm{L})^{\mathrm{c}}$ & $1.15(1.08-1.20)$ & $1.13(1.04-1.19)$ & $1.10(1.02-1.13)$ & $1.14(1.07-1.20)$ \\
\hline Bicarbonate $(\mathrm{mmol} / \mathrm{L})^{d}$ & $22.0(18.0-25.0)$ & $22.0(18.0-25.0)$ & $20.0(18.0-24.0)$ & $22.0(\mid 8.0-25.0)$ \\
\hline Potassium $(\mathrm{mmol} / \mathrm{L})^{e}$ & $3.8(3.4-4.2)$ & $3.8(3.3-4.3)$ & $3.9(3.6-4.1)$ & $3.8(3.4-4.2)$ \\
\hline$I_{N R}^{f}$ & $1.0(1.0-1.2)$ & I.I (I.0-I.2) & I.I (I.I-I.2) & $1.0(1.0-1.2)$ \\
\hline Platelet count $\left(\times 10^{9} / \mathrm{L}\right)^{g}$ & $213(166-270)$ & $206(148.5-254)$ & $203(15 \mathrm{I}-254)$ & $212(16 \mid-267)$ \\
\hline \multicolumn{5}{|l|}{$\begin{array}{l}\text { Medical treatment upon admission; } \\
\mathrm{N}(\%)\end{array}$} \\
\hline Diuretics & $300(36.2)$ & $65(40.4)$ & I3 (34.2) & $378(36.8)$ \\
\hline Metformin & $63(7.6)$ & $10(6.2)$ & I (2.6) & $74(7.2)$ \\
\hline Statins & $215(26.0)$ & $43(26.7)$ & $8(2 I . I)$ & $266(25.9)$ \\
\hline ACE-I/AT2A & $269(32.5)$ & $5 I(3 \mid .7)$ & $10(26.3)$ & $330(22.4)$ \\
\hline NSAID & $182(22.0)$ & $39(24.2)$ & $9(23.7)$ & $230(29.8)$ \\
\hline Acetaminophen & $25 I(30.3)$ & $45(28.0)$ & $10(26.3)$ & $306(29.8)$ \\
\hline
\end{tabular}

Notes: ${ }^{a}$ The median of all outpatient creatinine values measured during the 12 months before the index hospitalization. ${ }^{b} 55.3 \%$ missing. ${ }^{c} 38.0 \%$ missing. ${ }^{d} 39.5 \%$ missing. ${ }^{\mathrm{e}} 4.2 \%$ missing. ${ }^{\mathrm{f}} \mathrm{l} 1.6 \%$ missing. ${ }^{8} 9.9 \%$ missing.

Abbreviations: ACE-I/AT2A, angiotensin-converting enzyme inhibitors/angiotensin-2 receptor antagonists; CCI, Charlson Comorbidity Index; INR, international normalized ratio; IQR, interquartile range; NSAID, non-steroidal anti-inflammatory drug.

excluded. If a patient had multiple valid rhabdomyolysis diagnoses, only the first was included in the study.

The baseline creatinine level was defined as the mean of all outpatient results in the period from 365 days prior to the index hospitalization to 7 days before the index hospitalization. The date of the first $\mathrm{CK}$ value $>1000 \mathrm{U} / \mathrm{L}$ registered within the first 3 days of hospitalization was defined as the index date.

We used the unique Danish civil registration number assigned to all Danish residents at birth or upon immigration to link individual patients to national health registries containing information on medical history, medical treatment, emigration, and date of death. ${ }^{10}$

\section{Registries}

Data on hospital history were obtained from the Danish National Registry of Patients (DNRP). ${ }^{11,12}$ Use of medications was determined from redeemed prescriptions in the year prior to the index hospitalization, as recorded in the Danish National Health Service Prescription Database. ${ }^{13}$ Information on vital 
status and date of death was obtained from the Danish Civil Registration System (CRS). ${ }^{10}$

\section{Acute Kidney Injury}

Kidney Disease improving Global Outcome (KDIGO) guidelines were used to evaluate AKI. ${ }^{14}$ AKI was defined as an increase in serum creatinine ( $\mathrm{SCr}) \geq 26.5 \mu \mathrm{mol} / \mathrm{L}$ $(0.3 \mathrm{mg} / \mathrm{dL})$ from baseline or a greater than 1.5 -fold increase from baseline.

\section{Comorbidities}

We obtained data from the DNRP to estimate comorbidity burden based on Charlson Comorbidity Index (CCI) scores. The CCI score was divided into three levels: low (score 0), moderate (scores 1-2), and high (score $3+$ ). ${ }^{15}$

\section{Statistical Analysis}

For each outcome, we followed patients from the index date until diagnosis of a specific outcome, death, end of the study period, emigration, or end of the 30-day followup period, whichever came first.

We computed the 30-day risk of AKI ( $\geq$ stage 1), RRT, and death according to three levels of CK (1000-5000 U/L; 5001-15,000 U/L; and >15,000 U/L), using the cumulative incidence method, treating death as a competing risk for the AKI and RRT outcomes. For each outcome, spline regression was applied to further assess the relationship between $\mathrm{CK}$ on a continuous scale and the outcomes of interest.

In each of the spline regressions, we adjusted for gender, age, CCI score, primary diagnosis at hospital discharge, surgery during the index admission, medication use (Table 1) up to 1 year prior to the index admission, and laboratory results for the baseline period and on the date of the index admission (Table 1). We included CK results using a restricted cubic spline with five knots placed at the $5,27.5,50,72.5$, and 95 percentiles of the index CK level, using the CK level of $3000 \mathrm{U} / \mathrm{L}$ as the reference.

We used multiple imputation with predictive mean matching to impute missing baseline values of laboratory tests (Table 1) in the regression analyses. ${ }^{16}$ Multiple imputation was done separately for each outcome spline regression using 100 imputed datasets. For the outcomes death and RRT, the imputation models included the following variables: Initial CK level, gender, age, CCI score, surgery during index hospitalization (yes/no), primary discharge diagnosis, medical treatment on admission, laboratory test results, outcome event indicator, and
Nelson-Aalen estimate of the cumulative baseline hazard at the time of event/censoring. When imputing laboratory test values for the analyses using AKI as an outcome, we did not include an event indicator or baseline cumulative hazard estimate in the models. The imputed baseline creatinine values were used to calculate a median baseline creatinine level, and this median value was used to define AKI outcomes. ${ }^{16}$

Analyses were performed using the statistical software package SAS, version 9.4 (SAS Institute, Inc., NC).

\section{Results}

\section{Study Population}

The study included 1027 patients aged 18 years or older, with a $\mathrm{CK}$ value $>1000 \mathrm{U} / \mathrm{L}$ at hospital admission (Figure 1). The patients were $58.2 \%$ male with a median age of 73.5 years.

\section{Baseline Characteristics}

Baseline characteristics are shown in Table 1 according to CK level. The median CK level was $2257 \mathrm{U} / \mathrm{L}$ (IQR=1404-3961 U/L), with 828 (80.6\%) patients having a CK value of $1000-5000 \mathrm{U} / \mathrm{L}, 161(15.7 \%)$ having a CK value of 5001-15,000 U/L, and $38(3.7 \%)$ having a $\mathrm{CK}$ value $>15,001 \mathrm{U} / \mathrm{L}$ (Table 1 ).

Baseline creatinine results were available for $44.7 \%$ of the patients. Creatinine values measured upon hospital arrival increased as the level of CK increased (Table 1).

\section{Risk of AKI, RRT, and Death}

The 30-day risk of AKI according to CK level was $42 \%$ (95\% $\mathrm{CI}=38-45 \%), 44 \% \quad(36-52 \%)$, and $74 \% \quad(95 \%$ $\mathrm{CI}=57-85 \%$ ), respectively, and the risk of RRT was 3\% $(95 \% \mathrm{CI}=2 \%-5 \%), 4 \%(95 \% \mathrm{CI}=2-7 \%)$, and $11 \%$ (3-$23 \%$ ), respectively (Table 2 ).

The 30-day risk of death was $17 \%(95 \% \mathrm{CI}=14-20 \%)$, $16 \%(95 \% \mathrm{CI}=11-22 \%)$, and $11 \%(95 \% \mathrm{CI}=3 \%-23 \%)$ according to the three CK levels, respectively (Table 2).

\section{Spline Regression}

The adjusted spline plots showed an association between CK level and the outcomes (Figure 2). The adjusted HRs of AKI and RRT increased while the HR of death decreased with increasing CK level.

\section{Discussion}

We found that a high initial CK level was associated with an increased risk of AKI. Increasing CK level was also 
Table 2 Cumulative Incidence (Risk) of Acute Kidney Injury, Renal Replacement Therapy, and Death Within 30 Days by Initial Level of Creatine Phosphokinase (U/L) in 1027 Patients with Rhabdomyolysis

\begin{tabular}{|c|c|c|c|}
\hline & $\begin{array}{l}1000-5000 \\
(\mathrm{U} / \mathrm{L}) \\
(\mathrm{N}=\mathbf{8 2 8})\end{array}$ & $\begin{array}{l}500|-| 5,000 \\
(U / L) \\
(N=16 I)\end{array}$ & $\begin{array}{l}|5,00|+ \\
(U / L) \\
(N=38)\end{array}$ \\
\hline \multicolumn{4}{|c|}{ Outcome $(95 \% \mathrm{Cl})$} \\
\hline AKI $(n=454)$ & $42 \%(38-45)$ & $44 \%(36-52)$ & $74 \%(57-85)$ \\
\hline RRT $(n=37)$ & $3 \%(2-5)$ & $4 \%(2-7)$ & $11 \%(3-23)$ \\
\hline Death $(n=168)$ & $17 \%(14-20)$ & $16 \%(11-22)$ & $11 \%(3-23)$ \\
\hline
\end{tabular}

Abbreviations: $\mathrm{AKI}$, acute kidney injury; $\mathrm{Cl}$, confidence interval; $\mathrm{RRT}$, renal replacement therapy.

associated with increased risk of RRT, but decreased risk of death. However, the risk estimates of RRT and death were imprecise.

Our findings for the incidence (risk) of AKI are in the wide range found in other studies (from $4.7 \%$ to $94 \%)^{2,6-9,17-23}$ This range can be explained by use of different definitions for AKI and RM and different cut-off points for $\mathrm{CK}$ categories. Also, the studies had different designs, settings, and sample sizes; included a range of comorbid conditions; and their participants had different causes of $\mathrm{RM}^{8,24}$ In general, these study differences make their results difficult to compare. However, in one retrospective cohort study of 30-day outcomes that resembles our study (with AKI defined in accordance with the KDIGO criteria), AKI occurred in $38 \%$ of $400 \mathrm{RM}$ patients with $\mathrm{CK}$ values $>1000$ U/L. ${ }^{9}$ In our study, the 30-day risk of acute kidney injury according to the three creatinine phosphokinase levels was $42 \%(95 \% \mathrm{CI}=38-45 \%), 44 \%(36-52 \%)$, and $74 \%(95 \%$ $\mathrm{CI}=57-85 \%)$, respectively.

Whether $\mathrm{CK}$ is a prognostic factor for AKI remains controversial. While CK was found to have limited or no prognostic value in some studies, $6,7,9,17-21$ others found a low risk among patients whose $\mathrm{CK}$ level was less than 15,000-20,000 U/L. ${ }^{6}$ In a retrospective study of patients whose CK levels were in excess of $5000 \mathrm{U} /$ L, CK was independently associated with AKI. ${ }^{22}$ AKI has been observed among patients with $\mathrm{CK}$ values as low as $5000 \mathrm{U} / \mathrm{L}$, but usually in the setting of coexisting conditions such as sepsis, dehydration, and acidosis. ${ }^{6}$ A review and meta-analysis of the value of $\mathrm{CK}$ in predicting risk of RM-induced AKI identified a significant correlation between mean $\mathrm{CK}$ level and risk of crush-induced AKI. ${ }^{24}$

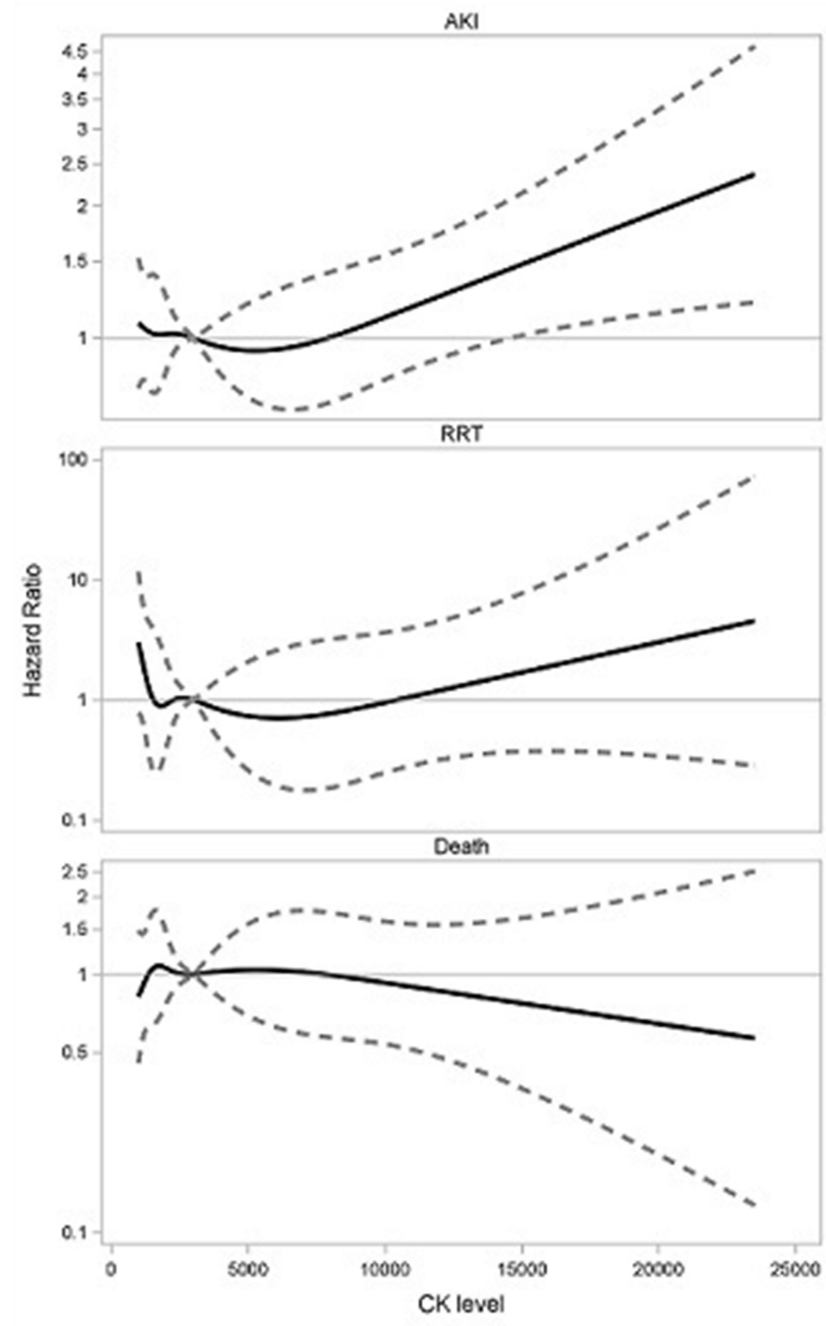

Figure 2 Restricted cubic spline plots of the association among creatine phosphokinase with acute kidney injury, renal replacement therapy, and death. Adjusted for gender, age, Charlson Comorbidlty Score, primary diagnosis at hospital discharge, surgery during the index admission, medication use up to I year prior to the index admission, and laboratory results for the baseline period and on the date of the index admission. Solid lines denote the hazard ratios. Dashed lines denote confidence intervals.

Abbreviations: AKI, acute kidney injury; CK, creatine phosphokinase; RRT, renal replacement therapy.

A study of a risk prediction score based on demographic, clinical, and laboratory variables at hospital admission found that $\mathrm{CK}$ levels alone were not sufficiently predictive of AKI. ${ }^{21}$

Relatively few patients in our study developed a need for treatment with RRT. Although our results indicated that patients with the highest $\mathrm{CK}$ levels are at increased risk of RRT, our estimates were imprecise and should be interpreted with caution.

In the literature, reported mortality rates in RM patients have ranged from $3.4 \%$ to $59 \%{ }^{6,9,17,19,21,22}$ Differences in study populations, incidence of kidney complications, 
coexisting comorbidities, triggering causes of RM, and follow-up time largely can explain this wide variation. Our results confirmed a study that found that $\mathrm{CK}$ was not associated with increased mortality. ${ }^{19}$

\section{Strengths and Limitations}

The method we used to identify RM patients ensured inclusion of a relatively large number of patients during a short period of hospital admissions, although the number of some of the outcomes is limited. It is fair to assume that CK values were measured among all hospitalized RM patients in the region, and thus that our study may be representative of RM patients. However, it remains possible that severely injured patients with RM were transferred directly to highly specialized trauma centers outside our region.

Another concern is our lack of accurate information on the causes for RM and the triggering mechanism involved in its pathophysiology. Depending on the severity of RM, these unknowns could affect prognosis.

We also lacked data on treatment of RM patients during hospitalization. Since early fluid resuscitation can reduce the risk of $\mathrm{AKI},{ }^{25}$ the time elapsed before $\mathrm{RM}$ detection, and delayed or insufficient treatment could have affected the outcome.

As well, we used the first value of $\mathrm{CK}>1000 \mathrm{U} / \mathrm{L}$ as the index value of $\mathrm{CK}$. It is possible that the maximum $\mathrm{CK}$ value during hospitalization would be more relevant. However, when we reanalyzed our data using maximum $\mathrm{CK}$ values, the relation between $\mathrm{CK}$ and outcomes did not change.

Lack of information on urine output may also be a limitation in our study. Alteration in urine flow has been shown to be a sensitive marker of AKI patients. ${ }^{26}$

Unmeasured confounding may also explain the finding of lower risk of death among patients in the group with highest CK-level. Younger men with low comorbidity score were more prominent in that group of patients. We do not have access to data on specific injuries (eg, excessive physical training) before admission and other relevant clinical information on health status, level of wellness, physical training habits, and socio-economic status with potential impact on survival. One may suggest that the group of patients with the highest level of CK values consisted of persons with some of these unmeasured baseline characteristics which may be associated with lower risk of death.

Finally, the numbers of outcomes were low. A larger sample size and more outcomes could have made our estimates more robust.

\section{Implications}

We found that $\mathrm{CK}$ is a risk factor for $\mathrm{AKI}$, a common complication of RM. In future studies that develop and test risk prediction models, $\mathrm{CK}$ should be included as an important factor in the prediction models. This could be helpful in identifying high-risk patients needing early intervention to prevent renal dysfunction.

\section{Conclusion}

Elevated initial creatine phosphokinase values were associated with an increased risk of acute kidney injury, while estimates of the risk of renal replacement therapy and death were imprecise.

\section{Abbreviations}

ACE-1, angiotensin-converting-enzyme inhibitor; AKI, acute kidney injury; AT2A, angiotensin 2 receptor antagonist; $\mathrm{CI}$, confidence interval; $\mathrm{CK}$, creatine phosphokinase; CCI, Charlson Comorbidity Index; CRS, civil registration system; DNRP, Danish National Registry of Patients; INR, international normalized ratio; IQR, interquartile range; KDIGO, Kidney Disease: Improving Global Outcomes; NSAID, non-steroidal anti-inflammatory drug; RM, rhabdomyolysis; RRT, renal replacement therapy; Scr, serum creatinine.

\section{Data Sharing Statement}

The datasets used and analyzed during the current study are available from the corresponding author for reasonable requests.

\section{Ethics Approval and Consent to Participate}

The study was approved by the Danish Data Protection Agency (REG-74-2014). As this study was registry-based and did not involve contact with patients or any intervention, it was not necessary to obtain approval from the Danish Scientific Ethics Committee.

\section{Author Contributions}

All authors made substantial contributions to the conception and design, acquisition of data, or analysis and interpretation of data; took part in drafting the article or revising it critically for important intellectual content; gave final approval of the version to be published; and agree to be accountable for all aspects of the work. 


\section{Funding}

This project received financial support from the NaestvedSlagelse-Ringsted Research Foundation (03-10-2014), Inger Bonnéns Fond (02-03-2015), and Snedkermester Sophus Jacobsen \& Hustru Astrid Jacobsens Fond (2409-2015).

\section{Disclosure}

Finn Erland Nielsen reports grants from Naestved-Slagelse -Ringsted Research Foundation (03-10-2014), Inger Bonnéns Fond (02-03-2015), and Snedkermester Sophus Jacobsen \& Hustru Astrid Jacobsens Fond (24-09-2015), during the conduct of the study. The authors report no other potential conflicts of interest for this work.

\section{References}

1. Nance JR, Mammen AL. Diagnostic evaluation of rhabdomyolysis. Muscle Nerve. 2015;51(6):793-810. doi:10.1002/mus.24606

2. Torres PA, Helmstetter JA, Kaye AM, Kaye AD. Rhabdomyolysis: pathogenesis, diagnosis, and treatment. Ochsner J. 2015;15:58-69.

3. Petejova N, Martinek A. Acute kidney injury due to rhabdomyolysis and renal replacement therapy: a critical review. Critical Care. 2014;18:224-231. doi:10.1186/cc13897

4. Shapiro ML, Baldea A, Luchette FA. Rhabdomyolysis in the Intensive Care Unit. J Intensive Care Med. 2012;27(6):335-342. doi: $10.1177 / 0885066611402150$

5. Cervellin G, Comelli I, Lippi G. Rhabdomyolysis: historical background, clinical, diagnostic and therapeutic features. Clin Chem Lab Med. 2010;48(6):749-756. doi:10.1515/CCLM.2010.151

6. Bosch X, Poch E, Grau JM. Rhabdomyolysis and acute kidney injury. N Engl J Med. 2009;361:62-72. doi:10.1056/NEJMra0801327

7. Bagley WH, Yang H, Shah KH. Rhabdomyolysis. Intern Emerg Med. 2011;11(3):210-218. doi:10.1007/s11739-007-0060-8

8. Scharman E, Troutman WG. Prevention of kidney injury following rhabdomyolysis: a systematic review. Ann Pharmacother. 2013;47:90-105. doi:10.1345/aph.1R215

9. Grunau BE, Pourvali R, Wiens MO, et al. Characteristics and thirty-day outcomes of emergency department patients with elevated creatine kinase. Acad Emerg Med. 2014;21:631-636. doi:10.1111/acem.12385

10. Schmidt M, Pedersen L, Sørensen HT. The Danish civil registration system as a tool in epidemiology. Eur J Epidemiol. 2014;29 (8):541-549. doi:10.1007/s10654-014-9930-3

11. Andersen TF, Madsen M, Jorgensen J, Mellemkjoer L, Olsen JH. The Danish national hospital register. A valuable source of data for modern health sciences. Dan Med Bull. 1999;46:263-268.

12. Nickelsen TN. [Data validity and coverage in the Danish national health registry. A literature review]. Ugeskr Laeger. 2001;164:33-37. Danish.

Clinical Epidemiology

\section{Publish your work in this journal}

Clinical Epidemiology is an international, peer-reviewed, open access, online journal focusing on disease and drug epidemiology, identification of risk factors and screening procedures to develop optimal preventative initiatives and programs. Specific topics include: diagnosis, prognosis, treatment, screening, prevention, risk factor modification,

Submit your manuscript here: https://www.dovepress.com/clinical-epidemiology-journal
13. Johannesdottir SA, Horváth-Puhó E, Ehrenstein V, Schmidt M, Pedersen L, Sørensen H. Existing data sources for clinical epidemiology: the Danish national database of reimbursed prescriptions. Clin Epidemiol. 2012;4:303-313. doi:10.2147/CLEP.S37587

14. Kidney Disease: Improving Global Outcomes (KDIGO) Acute Kidney Injury Work Group. KDIGO clinical practice guideline for acute kidney injury. Kidney Int. 2012;2:1.

15. Thygesen SK, Christiansen CF, Christensen S, Lash TL, Sorensen HT. The predictive value of ICD-10 diagnostic coding used to assess Charlson comorbidity index conditions in the population-based Danish national registry of patients. BMC Med Res Methodol. 2011;11:83. doi:10.1186/1471-2288-11-83

16. Siew ED, Peterson JF, Eden SK, Moons KG, Ikizler TA, Matheny ME. Use of multiple imputation method to improve estimation of missing baseline serum creatinine in acute kidney injury research. Clin J Am Soc Nephrol. 2013;8(1):10-18. doi:10.2215/CJN.00200112

17. Vangstad M, Bjornaas MA, Jacobsen D. Rhabdomyolysis: a10-year retrospective study of patients treated in a medical department. Eur J Emerg Med. 2017;26(3):199-204.

18. Simpson JP, Taylor A, Sudhan N, Menon DK, Lavinio A. Rhabdomyolysis and acute kidney injury: creatine kinase as a prognostic marker and validation of the McMahon score in a 10-year cohort. Eur J Anaesthesiol. 2016;33:906-912. doi:10.1097/ EJA.0000000000000490

19. Baeza-Trinidad R, Brea-Hernando A, Morera-Rodriquez S, et al. Creatinine as predictor value of mortality and acute kidney injury in rhabdomyolysis. Intern Med $J$. 2015;45(11):1173-1178. doi:10.1111/imj.12815

20. Chen C-Y, Lin Y-R, Zhao -L-L, Yang W-C, Chang Y-J, Wu H-P. Clinical factors in predicting acute renal failure caused by rhabdomyolysis in the ED. Am J Emerg Med. 2013;31(7):1062-1066. doi:10.1016/j.ajem.2013.03.047

21. McMahon GM, Zeng X, Waikar SS. A risk prediction score for kidney failure or mortality in rhabdomyolysis. JAMA Intern Med. 2013;173(19):1821-1828. doi:10.1001/jamainternmed.2013.9774

22. Rodriquez E, Soler MJ, Rap O, Barrios C, Orfila MA, Pascual J. Risk factors for acute kidney injury in severe rhabdomyolysis. PLoS One. 2013;8(12):1-6.

23. Wongrakpanich S, Kallis C, Prasad P, Rangaswami J, Rosenzweig A. The study of rhabdomyolysis in the elderly: an epidemiological study and single center experience. Aging Dis. 2018;9(1):1-7. doi:10.14336/AD.2017.0304

24. Safari S, Yousefifard M, Hashemi B, et al. The value of serum creatine kinase in predicting the risk of rhabdomyolysis-induced acute kidney injury: a systematic review and meta-analysis. Clin Exp Nephrol. 2016;20(2):153-161. doi:10.1007/s10157-015-1204-1

25. Better OS, Abassi ZA. Perspectives. Early fluid resuscitation in patients with rhabdomyolysis. Nat Rev Nephrol. 2011;7:416-422. doi:10.1038/nrneph.2011.56

26. Macedo E, Malhotra R, Claure-Del Granado R, Fedullo P, Mehta RL. Defining urine output criterion for acute kidney injury in critically ill patients. Nephrol Dial Transplant. 2011;26:509-515. doi:10.1093/ ndt/gfq332 systematic reviews, risk \& safety of medical interventions, epidemiology \& biostatistical methods, and evaluation of guidelines, translational medicine, health policies \& economic evaluations. The manuscript management system is completely online and includes a very quick and fair peer-review system, which is all easy to use. 